\title{
RESEARCH NOTES
}

\section{A NEW PROOF OF A THEOREM ABOUT GENERALIZED ORTHOGONAL POLYNOMIALS}

\author{
A.MCD. MERCER \\ Department of Mathematics and Statistics \\ University of Guelph \\ Ontario, Canada \\ (Received November 21, 1989)
}

\begin{abstract}
In this note it is shown that a fairly recent result on the asymptotic distribution of the zeros of generalized polynomials can be deduced from an old theorem of G. Polya, using a minimum of orthogonal polynomial theory.
\end{abstract}

KEY WORDS AND PHRASES. Toeplitz matrices, orthogonal polynomials, asymptotic zero distribution. MATHEMATICAL REVIEWS SUBJECT CLASSIFICATION. 15 A 18, 26 C 10.

\section{INTRODUCTION.}

Let $\alpha(x)$ be a distribution function [1] which has associated with it the unique sequence of polynomials $p_{n}(x)(n=0,1,2, \ldots)$ satisfying

$$
p_{n}(x)=\gamma_{n} x^{n}+\ldots\left(\gamma_{n}>0\right)
$$

and

$$
\int_{-\infty}^{+\infty} p_{m}(x) p_{n}(x) d \alpha(x)=\delta_{m, n}(m, n=0,1,2, \ldots)
$$

These polynomials will satisfy the recurrence relation:

$$
\left.\begin{array}{l}
x p_{n}(x)=\frac{\gamma_{n}}{\gamma_{n+1}} p_{n+1}(x)+\alpha_{n} p_{n}(x)+\frac{\gamma_{n-1}}{\gamma_{n}} p_{n-1}(x) \\
p_{-1}=\gamma_{-1}=0, \quad p_{0}(x)=\gamma_{0}, \quad \alpha_{n} \in \Re, \quad \gamma_{n}>0(n=0,1,2, \ldots)
\end{array}\right\}
$$

Conversely, a theorem due to Favard [2] states that if a sequence of polynomials satisfies (1.2) then a distribution function $\alpha(x)$ exists such that (1.1) holds. It is also known that the function $\alpha(x)$ is essentially unique [3] whenever the sequences $\left\{\frac{\gamma_{n}}{\gamma_{n+1}}\right\}$ and $\left\{\alpha_{n}\right\}$ are both bounded and that a necessary and sufficient condition for this to be the case is that the support of $d \alpha$,

$$
\operatorname{supp}(d \alpha)=\{x: \alpha(x-\varepsilon)<\alpha(x+\varepsilon), \forall \varepsilon>0\}
$$

is compact.

Suppose now that in (1.2) $\alpha_{n} \rightarrow \mathrm{a}$ and $\frac{\gamma_{n}}{\gamma_{n+1}} \rightarrow \frac{b}{2}$. Then one writes $\alpha \in M(\mathrm{a}, b)$ and if $b>0$ there 
will be no loss of generality in supposing that $\alpha \in M(0,1)[4]$, and we shall take this to be the case in all that follows. We now have $\operatorname{supp}(d \alpha)$ as a compact set and we will denote by $\Delta$ the smallest compact interval containing it. To elucidate the nature of $\operatorname{supp}(d \alpha)$ we quote the following lemma [4].

LEMMA A. Let $\alpha \in M(0,1)$ then $\operatorname{supp}(d \alpha)$ can be written as $\operatorname{supp}(d \alpha)-[-1,+1] \cup B$ where $B$ is enumerable and bounded and the only possible limit points of $B$ are \pm 1 . Furthermore, if $X$ is the set of all of the zeros of all the $p_{n}(x)$, then all of the limit points of $X$ belong to $\operatorname{supp}(d \alpha)$.

Once the polynomials $p_{n}(x)$ have been obtained, one can take a real-valued function $f$ defined on $\operatorname{supp}(d \alpha)$ and suitably restricted, and form the Toeplitz matrix

$$
H_{n}(f)=\left[\int_{\alpha} f(x) p_{i}(x) p_{j}(x) d \alpha(x)\right] \quad(i, j=0,1, \ldots, n-1)
$$

We shall denote the eigenvalues of this symmetric matrix, taken in ascending order, by $x_{k, n}(f)(1 \leq k \leq n)$. The following important theorem, with slight weaker hypotheses than are used here, has been proved by P. Nevai in [4]

THEOREM A. Let $\alpha \in M(0,1)$. Let $f$ be real-valued and $f \in C(\operatorname{supp}(d \alpha))$. Let $\Gamma$ be a compact interval containing $f(\operatorname{supp}(d \alpha)$ and suppose that $F \in C(\Gamma)$. Then, as $n \rightarrow \infty$

$$
\frac{1}{n} \sum_{k=1}^{n} F\left(x_{k, n}(f)\right) \rightarrow \frac{1}{\pi} \int_{-1}^{+1} \frac{F(f(t))}{\sqrt{1-t^{2}}} d t
$$

NOTE. It is not difficult to see that if $m$ and $M$ denote the lower and upper bounds of $f$ on $\operatorname{supp}(d \alpha)$ then

$$
m \leq x_{k, n}(f) \leq M \quad(1 \leq k \leq n: n=1,2, \ldots)
$$

We complete this introduction by quoting a theorem which we call Theorem B. It is a special case of a theorem appearing in [5] (see Section 5.2 there).

THEOREM B. Let $f_{1} \in C[-1,+1]$ be real-valued and write $g_{1}(\theta)=f_{1}(\cos \theta)$. Form the symmetric matrix

$$
K_{n}\left(f_{1}\right)=\left[\frac{1}{\pi} \int_{0}^{\pi} g_{1}(\theta) \cos (i-j) \theta d \theta\right] \quad(i, j=0,1, \ldots, n-1)
$$

Denoting by $m_{1}$ and $M_{1}$ the lower and upper bounds of $f_{1}$, let $F_{1} \in C\left[m_{1}, M_{1}\right]$. Then as $n \rightarrow \infty$ we have

$$
\frac{1}{n} \sum_{k=1}^{n} F_{1}\left(\lambda_{k, n}\left(f_{1}\right)\right) \rightarrow \frac{1}{\pi} \int_{0}^{\pi} F_{1}\left(g_{1}(\theta)\right) d \theta
$$

In this $\lambda_{k, n}\left(f_{1}\right)$ are the eigenvalues of $K_{n}\left(f_{1}\right)$ (all of which lie in $\left[m_{1}, M_{1}\right]$ ).

The purpose of this note is to give an alternative proof of Theorem A, showing that it may be deduced from Theorem B using two auxiliary lemmas.

\section{AUXILIARY LEMMAS}

LEMMA B. Let $\alpha \in M(0,1)$ and let $l$ be a fixed non-negative integer. Let $f \in C(\operatorname{supp}(d \alpha))$. Then as $n \rightarrow \infty$

$$
\int_{\alpha} f(x) p_{n}(x) p_{n+l}(x) d \alpha(x) \rightarrow \frac{1}{\pi} \int_{-1}^{+1} f(t) \frac{T_{l}(t) d t}{\sqrt{1-t^{2}}}
$$


where $T_{l}(t)$ is the Chebychev polynomial.

LEMMA C. Let $A \equiv\left[\mathrm{a}_{t, j}\right], B \equiv\left[b_{i, j}\right](i, j=0,1, \ldots)$ be Hermitian matrices whose principal $n \times n$ sections have eigenvalues

$$
\alpha_{1, n} \leq \alpha_{2, n} \leq \ldots \alpha_{n, n}
$$

and

$$
\beta_{1, n} \leq \beta_{2, n} \leq \ldots \leq \beta_{n, n}
$$

For all $n$ let all of these eigenvalues lie in a compact interval $\left[m_{2}, M_{2}\right]$. Let $k(n)(n=1,2, \ldots)$ be non-negative integers such that $k(n)=o(n)$ and let

$$
\left\|A_{n}^{(k)}-B_{n}^{(k)}\right\| \rightarrow 0 \text { as } n \rightarrow \infty
$$

where $A_{n}^{(k)}$, for example, denotes the matrix $\left[\mathrm{a}_{i, j}\right](i, j=k, k+1, \ldots, k+n-1)$ and $\|\cdot\|$ is any matrix norm. Then as $n \rightarrow \infty$ we have

$$
\frac{1}{n} \sum_{k=1}^{n} F_{2}\left(\alpha_{k, n}\right)-\frac{1}{n} \sum_{k=1}^{n} F_{2}\left(\beta_{k, n}\right) \rightarrow 0
$$

for any $F_{2} \in C\left[m_{2}, M_{2}\right]$.

Proofs of Lemma B are to be found in [4] and [6] while Lemma $C$ is a special case of a result proved in [7].

\section{MAIN RESULTS.}

We now proceed to prove our result, namely,

THEOREM 1. Theorem B implies Theorem A.

PROOF. $f \in C(\operatorname{supp}(d \alpha))$ and $\operatorname{supp}(d \alpha)$ is closed so we can extend the definition of $f$ to obtain $f_{+} \in C(\Delta)$ and this can be done in such a way that the bounds of $f_{+}$are also $m$ and $M$. Next, a polynomial $q$ can be found so that $\operatorname{Sup}_{\Delta}\left|f_{+}(x)-q(x)\right|$ is as small as we please and the bounds of $q$ on $\Delta$ are also $m$ and $M$. We shall prove the theorem, in the first instance, for such a polynomial $q$. The virtue of working with $q$ instead of the original $f$ lies in the fact that the two matrices which appear in Theorems A and B will, then, each be banded. This makes Lemma $C$ easy to apply.

Since the bounds of $q$ are the same as those given originally for $f$, we note that $\Gamma \supset q(\Delta)$. As in Theorem A let $F \in C(\Gamma)$. Now in Theorem B take $f_{1}$ to be $q$ and $F_{1}$ to be $F$. In the notation of that theorem $m_{1}=\inf _{[-1,+1]} q, M_{1}=\sup _{[-1,+1]} q$ and since $[-1,+1] \subset \Delta$ then $F \in C\left[m_{1}, M_{1}\right]$ (since $\left.\left[m_{1}, M_{1}\right] \subset[m, M]\right)$.

We deduce from Theorem B that

$$
\frac{1}{n} \sum_{k=1}^{n} F\left(\lambda_{k, n}(q)\right) \rightarrow \frac{1}{\pi} \int_{0}^{\pi} F\left(g_{2}(\theta)\right) d \theta
$$

where $g_{2}(\theta)=q(\cos \theta)$ and in which $\lambda_{k, n}(q)$ are the eigenvalues of

$$
\begin{aligned}
K_{n}(q) & =\left[\frac{1}{\pi} \int_{0}^{\pi} g_{2}(\theta) \cos (i-j) \theta d \theta\right] \\
& =\left[\frac{1}{\pi} \int_{-1}^{+1} q(t) T_{|i-j|}(t) \frac{d t}{\sqrt{1-t^{2}}}\right] \quad(i, j=0,1,2, \ldots, n-1)
\end{aligned}
$$


We next compare the infinite matrices $K_{\infty}(q)$ and

$$
H_{\infty}(q) \equiv\left[\int_{\partial} q(x) p_{\imath}(x) p_{j}(x) d \alpha(x)\right] \quad(i, j=0,1,2, \ldots)
$$

and we remark, first, that each is banded with bandwidth

$$
2(\text { degree } q)+1=N \quad \text { (say) }
$$

We next note that, according to Lemma $\mathrm{B}$,

$$
\int_{\Omega} q(x) p_{i}(x) p_{j}(x) d \alpha(x)-\frac{1}{\pi} \int_{-1}^{+1} q(x) T_{|i-j|}(x) \frac{d x}{\sqrt{1-x^{2}}} \rightarrow 0
$$

as $i \rightarrow \infty$ for each fixed $|i-j|=0,1,2, \ldots, N$. To apply Lemma $C$ we take $k(n)=[\sqrt{n}]$ and the matrix norm to be $\|\cdot\|_{\infty}$. According to (3.2) we will have

$$
\left\|K_{n}^{(k)}(q)-H_{n}^{(k)}(q)\right\|_{\infty} \rightarrow 0 \quad \text { as } n \rightarrow \infty
$$

and so we conclude that

$$
\frac{1}{n} \sum_{k=1}^{n} F\left(\lambda_{k, n}(q)\right)-\frac{1}{n} \sum_{k=1}^{n} F\left(x_{k, n}(q)\right) \rightarrow 0
$$

as $n \rightarrow \infty$. From (3.1) and (3.3) we deduce that, as $n \rightarrow \infty$

$$
\begin{aligned}
\frac{1}{n} \sum_{k=1}^{n} F\left(x_{k, n}(q)\right) & \rightarrow \frac{1}{\pi} \int_{0}^{\pi} F\left(g_{2}(\theta)\right) d \theta \\
& =\frac{1}{\pi} \int_{-1}^{+1} \frac{F(q(t))}{\sqrt{1-t^{2}}} d t
\end{aligned}
$$

which completes the proof of Theorem 1 for the polynomial case.

We can now extend (3.4) to the general case using the customary type of approximation argument. Let $\varepsilon>0$ be given. Then, by the uniform continuity of $F$ on $\Gamma$, there will be $\delta>0$ such that

$$
\left|F\left(\eta_{1}\right)-F\left(\eta_{2}\right)\right|<\varepsilon \quad \text { whenever } \quad\left|\eta_{1}-\eta_{2}\right|<\delta \quad\left(\eta_{1}, \eta_{2} \in \Gamma\right)
$$

With $f \in C(\operatorname{supp}(d \alpha))$ as given, we find the polynomial $q$ as previously described so that

$$
\left|f_{+}(t)-q(t)\right|<\delta \text { on } \Delta \text { and } m \leq q(t) \leq M \text { on }, \Delta
$$

We now have

$$
|F(f(t))-F(q(t))|<\varepsilon \text { on }[-1,+1]
$$

Next, consider the matrix

$$
H_{n}(f)=\left[\int_{\partial} f(x) p_{i}(x) p_{j}(x) d \alpha(x)\right] \quad(i, j=0,1, \ldots, n-1)
$$

and let $\|\cdot\|_{2}$ denote the spectral norm $(=\max \mid$ eigenvalues $\mid)$. Since

$$
\left|f_{+}(t)-q(t)\right|<\delta \text { on } \Delta
$$

and 


$$
\begin{aligned}
\left|x_{k, n}(q)-x_{k, n}(f)\right| & \leq\left\|H_{n}(q)-H_{n}(f)\right\|_{2} \quad(\text { see } \quad[8]) \\
& \leq \sup _{\text {supp }(d \alpha)}|q(t)-f(t)| \quad(\text { see } \quad \text { [4]) } \\
& \leq \sup _{\Delta}\left|q(t)-f_{+}(t)\right|<\delta
\end{aligned}
$$

we get

$$
\frac{1}{n}\left|\sum_{k=1}^{n}\left[F\left(x_{k, n}(q)\right)-F\left(x_{k, n}(f)\right)\right]\right|<\varepsilon
$$

The approximations (3.5) and (3.6), with $\varepsilon$ arbitrary, lead us, in the usual way, to deduce that (3.4) continues to hold when replaced by $f \in C(\operatorname{supp}(d \alpha))$. This completes the proof of Theorem 1 .

We remark, finally, that if in Theorem A we take the distribution function which yields the normalized Chebychev polynomials $\left\{T_{n}(x)\right\}_{0}^{\infty}$, then analysis similar to the above, but rather simpler, leads to the converse result that Theorem A implies Theorem B.

\section{REFERENCES}

1. G. Freud. "Orthogonal Polynomials," Pergamon Press, New York, 1971.

2. J. Favard. Sur les polynomes de Tchebicheff, C. R. Acad. Sci., Paris, 200 (1935), pp. 2052-2055.

3. T. S. Chihara. "An Introduction to Orthogonal Polynomials," Gordon and Breach, New York, 1978.

4. P. G. Nevai. "Orthogonal Polynomials," Mem. Amer. Math. Soc., Vol. 18, 1979.

5. U. Grenander and G. Polya. "Toeplitz Forms and their Applications," Univ. of Cal. Press, 1958.

6. G. Mitsis. "The Asymptotic Distribution of the Zeros of Generalized Orthogonal Polynomials, Master's Project, Univ .of Guelph, 1989.

7. A. McD. Mercer. On the Asymptotic Distribution of the Eigenvalues of Certain Hermitian Matrices, Jour. Approx. Theory (to appear).

8. Y. Ikebe, T. Inagaki and S. Miyamoto. The Monodromy Theorem, Cauchy's Interlace Theorem and the Courant-Fischer Theorem, Amer. Math. Monthly, Vol. 94, No. 4, pp. 852-854, April 1987. 


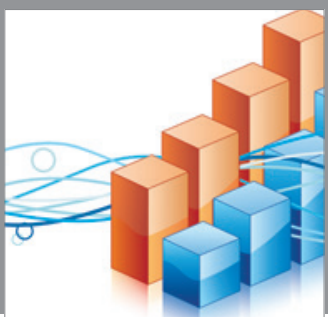

Advances in

Operations Research

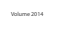

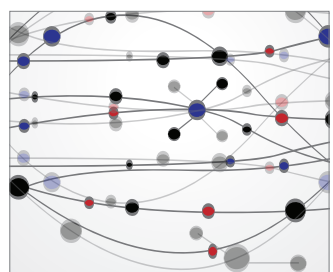

\section{The Scientific} World Journal
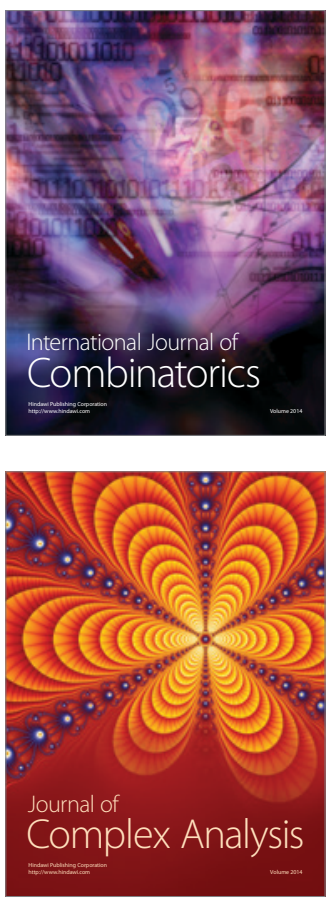

International Journal of

Mathematics and

Mathematical

Sciences
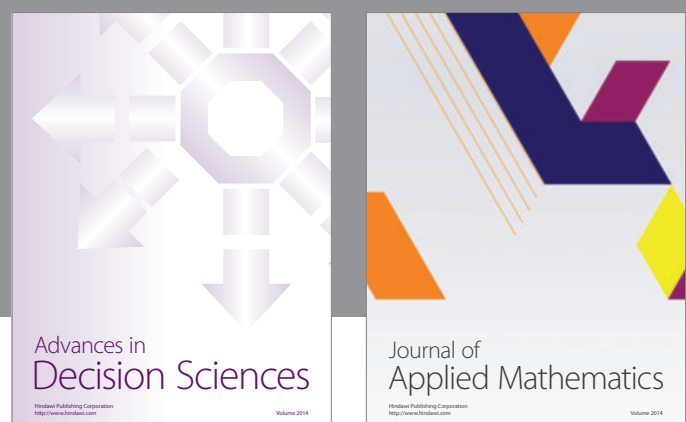

Journal of

Applied Mathematics
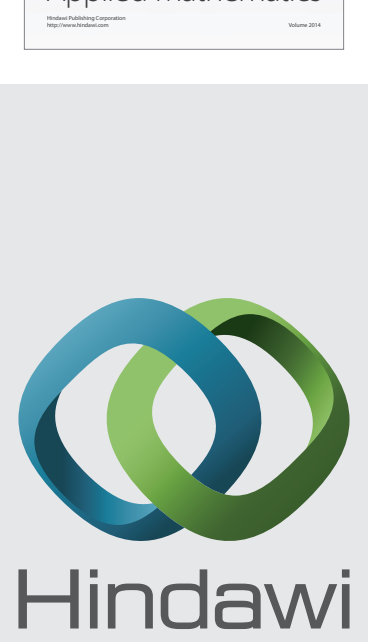

Submit your manuscripts at http://www.hindawi.com
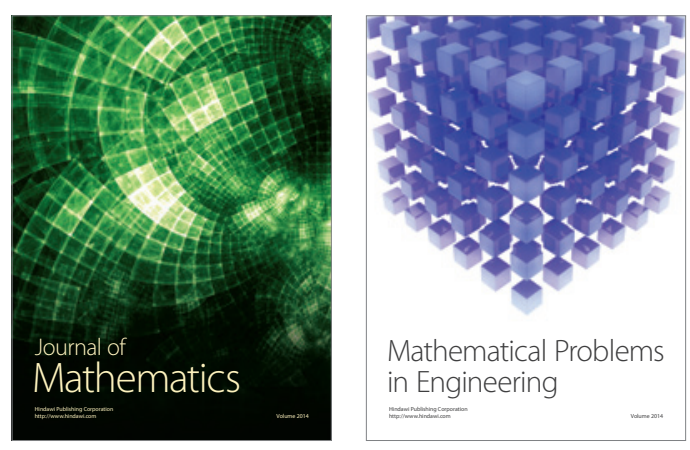

Mathematical Problems in Engineering
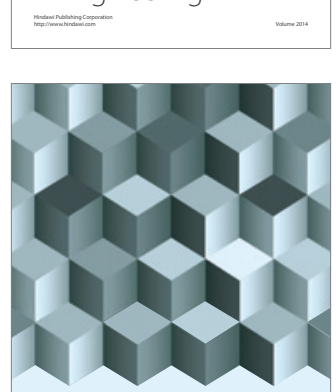

Journal of

Function Spaces
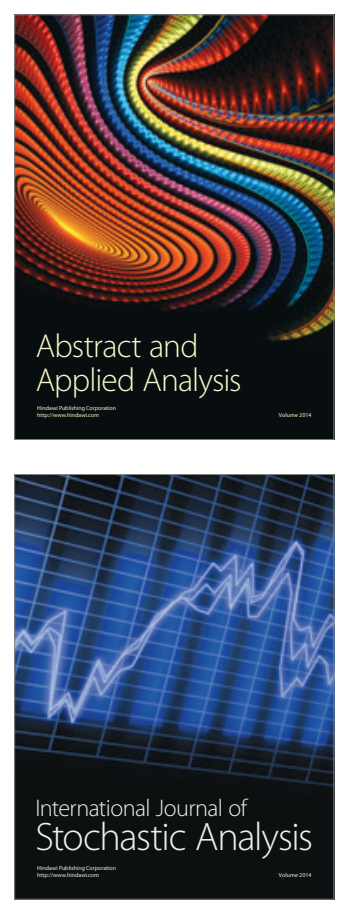

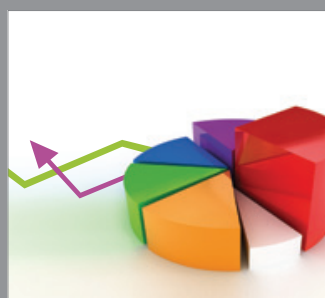

ournal of

Probability and Statistics

Promensencen
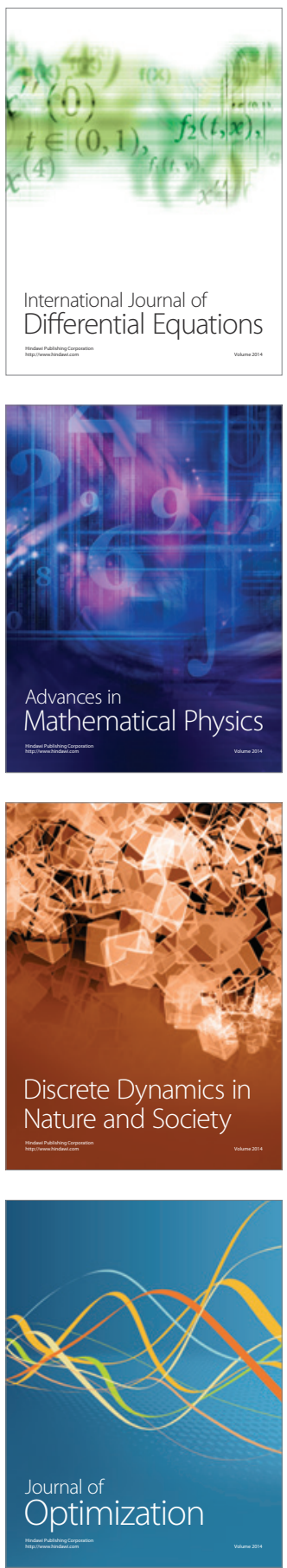